Competition between in-plane buckling and bending collapses in nanohoneycombs

This content has been downloaded from IOPscience. Please scroll down to see the full text. 2012 EPL 9816005

(http://iopscience.iop.org/0295-5075/98/1/16005)

View the table of contents for this issue, or go to the journal homepage for more

Download details:

IP Address: 89.202.245.164

This content was downloaded on 06/11/2013 at $11: 21$

Please note that terms and conditions apply. 


\title{
Competition between in-plane buckling and bending collapses in nanohoneycombs
}

\author{
Qiang Chen $^{1}$ and Nicola M. Pugno ${ }^{1,2,3(a)}$ \\ ${ }^{1}$ Laboratory of Bio-Inspired Nanomechanics "Giuseppe Maria Pugno", Department of Structural, Geotechnical \\ and Building Engineering, Politecnico di Torino - I-10129, Torino, Italy, EU \\ 2 National Institute of Nuclear Physics, National Laboratories of Frascati - Via E. Fermi 40, \\ I-00044, Frascati, Italy, EU \\ ${ }^{3}$ National Institute of Metrological Research - Strada delle Cacce 91, I-10135, Torino, Italy, EU
}

received 18 November 2011; accepted in final form 24 February 2012

published online 30 March 2012

PACS 62.20.M - Structural failure of materials

PACS 61.43.Gt - Powders, porous materials

PACS 61.46.-w-Structure of nanoscale materials

\begin{abstract}
The classical hexagonal honeycomb theory for the uniaxial loading case, developed by Gibson and Ashby, considers buckling of the cell walls parallel to one symmetry axis. In general, buckling may also occur in the cell walls inclined with respect to the two symmetry axes. Therefore, in this letter, under the uniaxial loading conditions, we derive the critical stresses of buckling and bending collapses of nanohoneycombs, for which the surface effect is included. Furthermore, the competition between the two failure modes is studied. The present theory could be used to design new nanoporous materials, e.g., scaffolds for the regenerative medicine or energy-absorption materials.
\end{abstract}

Copyright (c) EPLA, 2012

Introduction. - Porous materials, which can be often found in Nature [1], have high mechanical efficiency and are widely used as modern materials; for instance, they are used as core materials in sandwich structures [2], common in aerospace engineering, or as energy-absorption materials to reduce the loading impact and improve the resistance to crushing [3]. The mechanical properties of a honeycomb, and in particular its failure mechanisms, are therefore of great interest to material scientists. A number of pioneering studies [4-11] analyzed its in-plane and out-of-plane mechanical behaviors (e.g., elastic buckling); for the in-plane deformation mechanism, the stress-strain curve $[3,7,10]$ is described by the linear elastic, plastic plateau and densification regions. Lee et al. [12] studied the mechanical behavior and deformation mechanism of a honeycomb composite at the temperature ranging from $25^{\circ} \mathrm{C}$ to $300^{\circ} \mathrm{C}$. The classical approach was developed by Gibson and Ashby [10]. They systematically investigated elastic buckling, plastic and brittle collapses of the 2D hexagonal honeycombs for uniaxial and biaxial loading cases. In the uniaxial loading case, the classical prediction only considers the buckling of the cell walls parallel to one

(a) E-mail: nicola.pugno@polito.it symmetry axis; in spite of this, buckling could occur first in the inclined cell walls.

When the cell size comes down into the nanoscale, the surface effect, due to the high surface-to-volume ratio $[13,14]$, starts to play an important role in determining the mechanical properties of the structure. Extensive works [15-18] studied the influence of surface effect on linear elastic and plastic properties of nanomaterials.

Considering the above statements, here we develop a theory to determine the buckling competition between the inclined and parallel cell walls with respect to the symmetry axes (in the following text, we simply call them inclined and parallel cell wall, respectively) of nanohoneycombs, under the uniaxial loading condition. Moreover, we separately establish the criteria for the competition of failure modes between buckling and bending collapses in the two principal directions [19]. The present theory could be used to design new stronger and stiffer honeycomb nanomaterials and in general to tune their mechanical response.

The surface effect on Young's modulus and the buckling of inclined columns. -

The surface effect on Young's modulus. As the structural size reduces to nanoscale, the surface-to-volume 

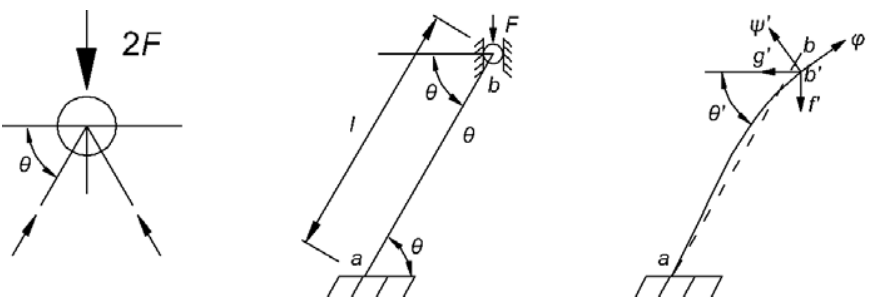

Fig. 1: Schematic of an inclined column.

ratio increases and the surface effect, induced by the surface atoms of the structure, dominates the mechanical behavior. A classical expression of surface stresses, basing on the energy method, has been derived for the linearelastic body [14]:

$$
\tau_{i j}=\tau_{i j}^{0}+S_{i j}: \varepsilon_{i j},
$$

where, $\tau_{i j}$ is the 2-rank surface stress tensor, $\tau_{i j}^{0}$ is the surface residual stress tensor, $S_{i j}$ is the surface stiffness matrix, $\varepsilon_{i j}$ is the surface strain tensor and the symbol ":" represents the inner product of tensors. For the onedimensional case, it can be expressed in a linear form [15]:

$$
\tau^{s}=\tau^{0}+E^{s} \varepsilon,
$$

where, $\tau^{s}$ is the surface stress, $\tau^{0}$ is the surface residual stress, $E^{s}$ is the surface Young's modulus and $\varepsilon$ is the surface strain.

To predict the effective Young's modulus $E^{e f f}$, we apply Miller and Shenoy's [20] approach:

$$
\frac{E^{e f f}}{E}=1+\frac{E^{s}}{E t}\left(6+2 \frac{t}{b}\right),
$$

where, $E$ is the Young's modulus without considering the surface effect, $E^{s}$ is the surface Young's modulus, whereas, $t$ and $b$ are the cross-sectional thickness and width of the cell wall, respectively.

Equation (3) obeys the simple linear scaling law [21] $E^{e f f} / E=1+\alpha l_{i n} / L, l_{\text {in }}$ representing a material intrinsic length, under which surface effect plays an important role; $\alpha$ is a dimensionless constant, which depends on the geometry of the structural elements and their deformations and $L \equiv t$. Note that the term in the bracket will be close to 6 if $t \ll b$ (plate) or 8 if $t=b$ (square beam).

The buckling of inclined columns. Basing on the large deflection theory, Chang [22] derived the buckling formulas of inclined columns (fig. 1) with different boundary conditions. A numerical factor $\beta$ reflects the boundary conditions and it is defined as

$$
\beta^{2}=-\varphi R^{2}
$$

with

$$
\varphi=-\left(f^{\prime} \sin \theta^{\prime}+g^{\prime} \cos \theta^{\prime}\right),
$$

where $R=l / r$ is the slenderness ratio; $f^{\prime}=F /(A E)$ and $g^{\prime}=f^{\prime} \cot \theta^{\prime}$ are constraint forces acting in the vertical

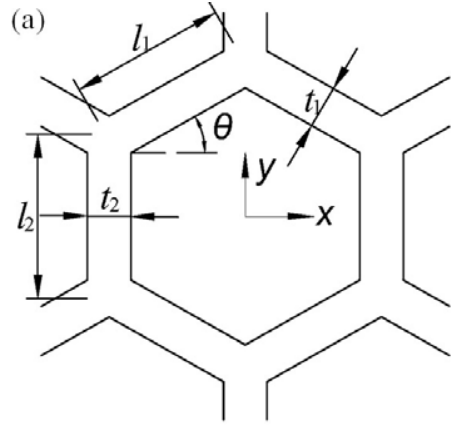

(d)

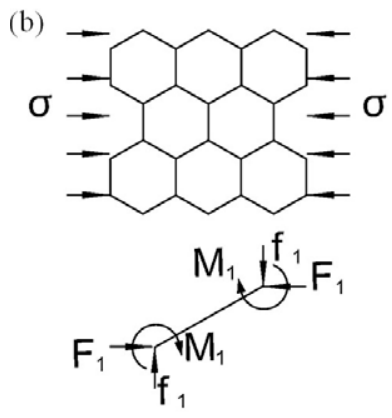

(c)
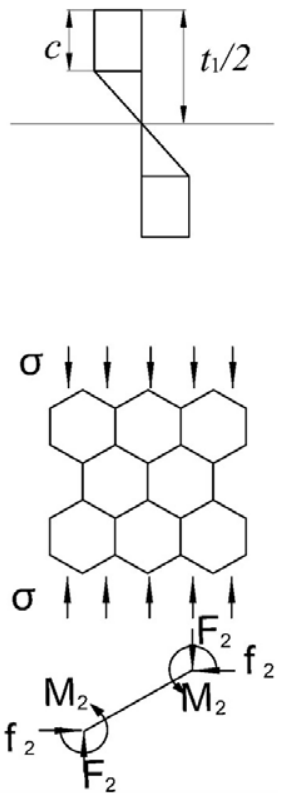

Fig. 2: (a) Geometrical parameters of a unit cell; (b) loading in the $x$-direction; (c) loading in the $y$-direction; (d) stress distribution on the cross-section of cell walls. The moments in each scheme are identical due to the lattice periodicity.

and horizontal directions at the deformable end, respectively; $\theta^{\prime}$ is the inclined angle made by the column and the horizontal line after the column's deformation; $l$ is the length of the column; $r$ is the radius of gyration; $F$ is the external force; $A$ is the cross-sectional area; $E$ is Young's modulus.

Considering the inclined columns as fixed at both ends, the eigenvalue equation with respect to $\beta$ is [22]:

$$
\frac{\beta^{2}}{R^{2}} \cot ^{2} \theta+2 \frac{1-\cos \beta}{\beta \sin \beta}-1=0 .
$$

For the sake of simplicity, when $50<R<500, \beta$ is considered as a constant and equal to 8.669 , because it has a minor change when $\theta$ varies in the range $15^{\circ}-75^{\circ}$. Note that $\beta=8.669$ (i.e., $\theta=15^{\circ}$ ) is conservative, compared with 8.986 when $\theta=75^{\circ}$. Rearranging eq. (4) and substituting $r=\sqrt{I / A}$ into it, we find the buckling load of the inclined columns at the initial state $\left(\theta^{\prime}=\theta\right)$ as

$$
F=\frac{\beta^{2} E I \sin \theta}{l^{2}},
$$

where $I$ is the cross-sectional moment of inertia.

\section{Failure analysis in the $x$-direction. -}

Buckling. As discussed before, the inclined cell walls are columns clamped at both ends. In the $x$-direction (fig. 2(b)), the area, on which the external stress acts, is $k_{1} b\left(l_{2}+l_{1} \sin \theta\right)$, where $k_{1}$ is the number of unit cells, $b$ is the width of the cell wall, $\theta$ is the angle between the inclined cell wall and the horizontal line, and $l_{2}, l_{1}$ are the 
lengths of the parallel and inclined cell walls, respectively. Therefore, the equivalent force acting on each inclined cell wall is $F_{1}=\sigma b\left(l_{2}+l_{1} \sin \theta\right)$, where $\sigma$ is the external stress acting on the unit cell. Thus, substituting $F_{1}$ into eq. (6) and considering the surface effect, the external critical buckling stress is calculated as

$$
\sigma_{c r, 1}^{b}=\frac{\beta^{2} E_{1}^{e f f}}{12} \frac{\cos \theta}{\alpha+\sin \theta}\left(\frac{t_{1}}{l_{1}}\right)^{3} \quad \text { with } \alpha=l_{2} / l_{1} .
$$

Competition between buckling and bending failure. The yield stress of the constituent material is denoted by $\sigma_{y s}$, and the elastic-plastic stress distribution is shown in fig. 2(d); then, the related failure moment due to bending is

$$
M_{\text {max }, p}=\sigma_{y s} b t_{1}^{2}\left[\frac{c}{t_{1}}\left(1-\frac{c}{t_{1}}\right)+\frac{2}{3}\left(\frac{1}{2}-\frac{c}{t_{1}}\right)^{2}\right] .
$$

According to Ashby and Gibson [4], the maximum bending moment $M_{\max , 1}=F_{1} l_{1} \sin \theta / 2$ of the cell wall is at its two ends. Then, the critical condition for the competition between elastic buckling and plastic collapse can be obtained by setting

$$
M_{\max , p}=M_{\max , 1} .
$$

Thus, we find the critical stress for plastic collapse:

$$
\sigma_{c r, 1}^{p}=\frac{2\left[\frac{c}{t_{1}}\left(1-\frac{c}{t_{1}}\right)+\frac{2}{3}\left(\frac{1}{2}-\frac{c}{t_{1}}\right)^{2}\right] \sigma_{y s}}{(\alpha+\sin \theta) \sin \theta}\left(\frac{t_{1}}{l_{1}}\right)^{2} .
$$

Accordingly, by comparing $\sigma_{c r, 1}^{b}=\sigma_{c r, 1}^{p}$, we find the criterion for the competition between buckling and bending collapses in the $x$-direction as

$$
\left(\frac{t_{1}}{l_{1}}\right)_{c r i t}=\frac{48\left[\frac{c}{t_{1}}\left(1-\frac{c}{t_{1}}\right)+\frac{2}{3}\left(\frac{1}{2}-\frac{c}{t_{1}}\right)^{2}\right]}{\beta^{2} \sin 2 \theta} \frac{\sigma_{y s}}{E_{1}^{e f f}} .
$$

Failure analysis in the $y$-direction. - In the $y$ direction (fig. 2(c)), since the buckling collapse may take place in both the inclined and parallel cell walls, the analysis is different from that for the $x$-direction. Here, we first consider the buckling competition between the two types of cell walls.

Buckling competition between the inclined and parallel cell walls. In this case, the inclined cell walls are treated as those in the $x$-direction. The area, on which the external stress acts, is $2 k_{2} b l_{1} \cos \theta$, where $k_{2}$ is the number of unit cells in the $y$-direction. Correspondingly, the number of cell walls is $2 k_{2}$, thus, the equivalent force acting on each inclined cell wall is $F_{2}=\sigma b l_{1} \cos \theta$ and the buckling stress is obtained as

$$
\sigma_{c r, 2}^{(1)}=\frac{\beta^{2} E_{1}^{e f f}}{12} \tan \theta\left(\frac{t_{1}}{l_{1}}\right)^{3}
$$

Regarding the critical buckling stress of the parallel cell walls, when $t_{1}=t_{2}$, the classical result yields [10]

$$
\sigma_{c r, 2}^{(2)}=\frac{(n \pi)^{2} E_{2}^{e f f}}{24} \frac{1}{\cos \theta} \alpha\left(\frac{t_{2}}{l_{2}}\right)^{3}
$$

where $n$, determined by $\alpha$, is a numerical factor and reflects the boundary conditions. The external buckling stress, in the $y$-direction, is the minimum between the buckling stresses of the inclined and parallel cell walls:

$$
\sigma_{c r, 2}^{b}=\min \left(\sigma_{c r, 2}^{(1)}, \sigma_{c r, 2}^{(2)}\right) .
$$

Competition between buckling and bending failure. Similarly, the maximum bending moment $M_{\max , 2}=F_{2} l_{1} \cos \theta / 2$ of the beam is at its two ends. Thus, the critical condition for the competition between elastic buckling and plastic collapse can be obtained through

$$
M_{\max , p}=M_{\max , 2}
$$

and the critical stress for plastic collapse is obtained as

$$
\sigma_{c r, 2}^{p}=\frac{2\left[\frac{c}{t_{1}}\left(1-\frac{c}{t_{1}}\right)+\frac{2}{3}\left(\frac{1}{2}-\frac{c}{t_{1}}\right)^{2}\right] \sigma_{y s}}{\cos ^{2} \theta}\left(\frac{t_{1}}{l_{1}}\right)^{2} .
$$

Then, the competition between buckling and bending collapses is described by setting $\sigma_{c r, 2}^{b}=\sigma_{c r, 2}^{p}$, i.e.,

$$
\left(\frac{t_{1}}{l_{1}}\right)_{c r i t}=\cos \theta \sqrt{\frac{\min \left(\sigma_{c r, 2}^{(1)}, \sigma_{c r, 2}^{(2)}\right)}{2\left[\frac{c}{t_{1}}\left(1-\frac{c}{t_{1}}\right)+\frac{2}{3}\left(\frac{1}{2}-\frac{c}{t_{1}}\right)^{2}\right] \sigma_{y s}}} .
$$

Analytic results. - Here, we employ aluminum as a case study; Young's modulus is $E=75 \mathrm{GPa}$, the surface Young's modulus is $E^{s}=2.45 \mathrm{~N} / \mathrm{m}$ [20], $\sigma_{y s}=4.3 \mathrm{GPa}[23], t_{1}=5 \mathrm{~nm}$ and $b \gg t_{1}$. Accordingly, we obtain $E_{1}^{e f f}=75.5 \mathrm{GPa}$. For the $x$-direction, the results of the parametric analysis are reported in fig. 3. Figure 3(a) shows that the critical buckling stress decreases as $\alpha$ increases, when the inclined angle $\theta$ is fixed. This is because the force acting on the inclined cell walls increases with increasing $\alpha$, under the same external stress, and thus a constant buckling force of the cell walls results in a smaller external stress for increasing $\alpha$. It also shows that the buckling stress decreases as the inclined angle $\theta$ increases, when $\alpha$ is fixed. As for the competition between buckling and bending collapses, the analytical result is reported in fig. 3(b); we can see that the criteria are symmetric with respect to $\theta=45^{\circ}$, for which the lowest critical value of $t_{1} / l_{1}$ emerges; also the load for fully plastic failure $(c=0)$ is higher than that for brittle failure $\left(c=t_{1} / 2\right)$. This is because fully plastic failure requires larger moments. Figure $3(\mathrm{c})$ provides a schematic of the failure mechanisms. 

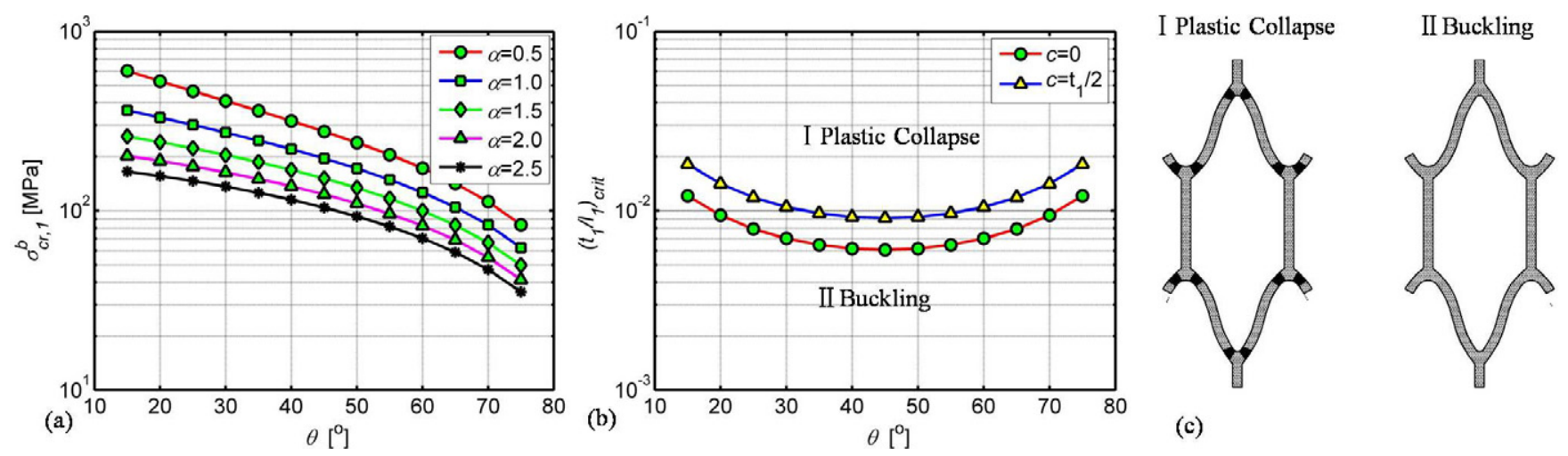

Fig. 3: (Colour on-line) (a) Critical buckling stress vs. $\theta$ influenced by $\alpha$, for when $t_{1} / l_{1}=0.1$; (b) critical value of $t_{1} / l_{1}$ for buckling and bending competing failures in the $x$-direction; (c) schematics of the failure modes reported in (b). Note that the black parts in (c) denote points subjected to bending failure.
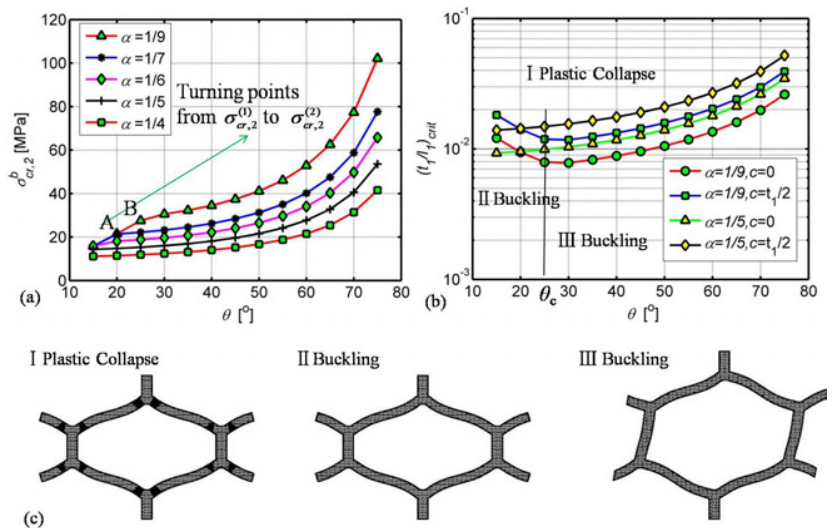

Fig. 4: (Colour on-line) (a) Buckling stresses $\sigma_{c r, 2}^{(1)}$ and $\sigma_{c r, 2}^{(2)}$ vs. $\theta$, as a function of $\alpha$; (b) critical value of $t_{1} / l_{1}$ for buckling and bending competing failures; (c) schematics of the failure modes for the case $\alpha=1 / 9$ reported in (b). Note that the black parts in (c) denote points subjected to bending failure.

If we consider $t_{1} / l_{1}=0.05, t_{1}=t_{2}=5 \mathrm{~nm}$, we find $E_{2}^{e f f}=E_{1}^{e f f}=75.5 \mathrm{GPa}$. For the $y$-direction, the analytic results of buckling stress and $t_{1} / l_{1}$ are reported in fig. 4 . Figure 4(a) shows that buckling of inclined cell walls takes place (for $t_{1}=t_{2}$ ) as the length ratio $\alpha$ decreases and the failure-transition points (from $\sigma_{c r, 2}^{(1)}$ to $\sigma_{c r, 2}^{(2)}$ ) move forwardly to large angles; in particular, when $\alpha=1 / 4$ or $1 / 5$, the buckling only occurs in the parallel cell walls. This is because $\sigma_{c r, 2}^{(1)}$ increases when $\theta$ increases and $\sigma_{c r, 2}^{(2)}$ increases when $\alpha$ decreases. Figure $4(\mathrm{~b})$ shows that decreasing $t_{1} / l_{1}$ leads to buckling. Besides, we can also see that if $\alpha=1 / 5$, the competition is only between the buckling of the parallel cell walls, as shown in fig. 4(a), and bending collapses of the inclined cell walls. For $\alpha=1 / 9$ the buckling of inclined cell walls gets involved, namely, the curve denotes a critical condition between the buckling of inclined cell walls and its bending collapses when $\theta<\theta_{c}$ and that between the buckling of parallel cell walls and bending collapses of the inclined cell walls when $\theta>\theta_{c}$. Figure 4(c) provides a schematic of the failure mechanisms $(\alpha=1 / 9)$.

Compared with the classical honeycomb theory, our analytical approach gives us an interesting result about the failure transition of a nanohoneycomb under the uniaxial loading case, governed by the parameter $t_{1} / l_{1}$. The derivation includes the surface effect, but it can be neglected when the cross-sectional size $t_{1}$ is larger than $\sim 100 \mathrm{~nm}$ [24].

Conclusions. - In this letter, under the uniaxial loading conditions, we have derived the buckling stress of the inclined cell walls in nanohoneycombs, which is not treated in the classical approach by Gibson and Ashby. Then, basing on the existing buckling and plasticfailure theory, the competition between these two failure mechanisms is studied, including the surface effect peculiar of the nanoscale.

The present theory could be used to design new nanoporous materials, e.g., scaffolds for the regenerative medicine or energy-absorption materials.

$$
* * *
$$

The research related to these results has received funding from the European Rearch Council under the European Union's Seventh Framework Programme (FP7/2007-2013)/ERC Grant agreement No. 279985 BIHSNAM.

\section{REFERENCES}

[1] Seki Y, Schneider M. S. and Meyers M. A., Acta Mater., 53 (2011) 5281.

[2] Foo C. C., Chai G. B. and Seah L. K., Compos. Struct., 80 (2007) 588.

[3] Xue Z. Y. and Hutchinson J. W., Int. J. Numer. Methods Eng., 65 (2006) 2221. 
[4] Gibson L. J. and Ashby M. F., Proc. R. Soc. London, Ser. A, 382 (1982) 25.

[5] Warren W. E. and Kraynik A. M., Mech. Mater., 6 (1987) 27.

[6] Zhang J. and Ashby M. F., Int. J. Mech. Sci., 34 (1992) 491.

[7] Papka S. D. and Kyriakides S., J. Mech. Phys. Solids, 42 (1994) 1499.

[8] Papka S. D. and Kyriakides S., Int. J. Solids Struct., 35 (1998) 239.

[9] Papka S.D. and Kyriakides S., Acta Mater., 46 (1998) 2765.

[10] Gibson L. J. and Ashby M. F., Cellular Solids: Structure and Properties, 2nd edition (Cambridge University Press, Cambridge) 1997.

[11] Bhat B. T. and Wang T. G., J. Mater. Sci., 25 (1990) 5157.

[12] LeE H. S., Hong S. H., LeE J. R. and Kim Y. K., J. Mater. Sci., 37 (2002) 1265.

[13] Pavese M., Musso S. and Pugno N., J. Nanosci. Nanotechnol., 10 (2010) 4240.

[14] Cammarata R. C., Prog. Surf. Sci., 46 (1994) 1.
[15] Wang G. F. and Feng X. Q., Appl. Phys. Lett., 94 (2009) 141913.

[16] Shankar M. R. and King A. H., Appl. Phys. Lett., 90 (2007) 141907.

[17] Wong E. W., Sheehan P. E. and Lieber C. M., Science, 277 (1997) 1972.

[18] Zhang W. J., Wang T. J. and Chen X., Int. J. Plast., 26 (2010) 957.

[19] Peng B., Pugno N. and Espinosa H. D., Int. J. Solids Struct., 43 (2006) 3292.

[20] Miller R. E. and Shenoy V. B., Nanotechnology, 11 (2000) 139.

[21] Duan H. L., Wang J, Karihaloo B. L. and Huang Z. P., Acta Mater., 54 (2006) 2983.

[22] Chang C. H., Lect. Notes Appl. Comput. Mech., 22 (2005) 93.

[23] Pastor-Abia L., Caturla M. J., SanFabian E., Chiappe G. and Louis E., Phys. Status Solidi C, 6 (2009) 2119.

[24] Sohn Y. S., Park J., Yoon G., Song J., Jee S. W., Lee J. H., NA S., Kwon T. and EOM K., Nanoscale Res. Lett., 5 (2010) 211. 